\title{
Traumatic fibroma of the tongue -A treatment modality study
}

\section{S. Sakthivel ${ }^{1, *}$, C. Gokula Krishnan ${ }^{2}$, Manishaa. V' ${ }^{1}$ K. Sumithra ${ }^{1}$, S. Mukesh ${ }^{1}$, S. Gopikrishnan ${ }^{1}$}

${ }^{1}$ MDS OMFS, Velss Dental Care, Chennai, India

${ }^{2}$ MDS OMFS, C.R. Dental and Implant centre, Chennai, India

*Corresponding Author: V. S. Sakthivel, MDS OMFS, Velss Dental Care, Chennai, India

Received date: 20 May 2021; Accepted date: 25 May 2021; Published date: 28 May 2021

Citation: Sakthivel VS, Krishnan CG, Manishaa V, Sumithra K, Mukesh S, et al. Traumatic fibroma of the tongue - a treatment modality study. JMed Case Rep Case Series 2(4): https://doi.org/10.38207/jmcrcs20210060

Copyright: (C) 2021 V.S. Sakthivel. This is an open-access article distributed under the terms of the Creative Commons Attribution License, which permits unrestricted use, distribution, and reproduction in any medium, provided the original author and source are credited.

\begin{abstract}
Traumatic fibroma is one of the most common oral soft tissue lesions that occur secondaryto trauma. It is a benign hyperplastic reactive lesion. It can occur in any age group and at almost any soft tissue site. The growth may frequently cause hindrances in speech and mastication and may also cause esthetic impairments. This article discusses the clinical examination, diagnosis, investigations, and treatment of a case of traumatic fibroma in a male teenaged patient.
\end{abstract}

Keywords: Fibroma, trauma, tongue, reactive lesion

\section{Introduction}

The oral cavity is the most common site for localized reactive overgrowths. Oral fibroma, also known as Traumatic fibroma, also known as an irritational fibroma or focal fibrous hyperplasia, is a benign exophytic lesion that occurs because of injury. Fibroma is the most common benign soft-tissue neoplasm in the oral cavity [1]. It is essentially a fibrous submucosal mass that consists of granulation

\section{Case Report}

A twelve-year-old male patient reported to Vihaa Multispeciality Hospital, Annanagar, Chennai, India, with a chief complaint of swelling and discomfort in the posterior part of his tongue for the past two months. The patient further gave a history of a slow-growing swelling, that was small initially but increased in size with time. He informed us that the swelling caused disturbances in normal speech and swallowing of food. The patient also gavea history of traumatic bleeding of the tongue while toothbrushing two months ago. He did tissue and scar tissue [2]. Traumatic fibroma is the healed product of an inflammatory hyperplastic lesion [3]. Irregular sharp margins of teeth or bone, toothbrush trauma, iatrogenic trauma, dental appliances, or chronic biting are some of the common injury-causing agents. In this article, we report a case of Traumatic Fibroma of the posterior tongue.

nothave any significant medical, dental, or family history.

On intraoral examination, a solitary reddish-pink oval growth was seen in the posterior dorsal surface of the tongue at the junction of the anterior two-thirds and posterior one-third of the tongue (Figure 1). The growth was painless, sessile, and well-circumscribed with an irregular surface. It measured 5x5x3 mm approximately, was firm in consistency, and non-tender upon palpation.

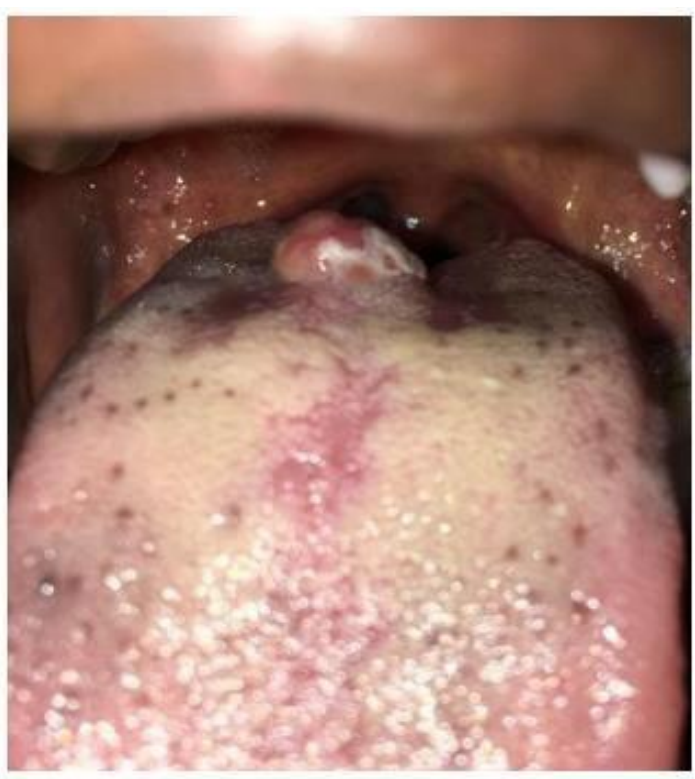

Figure 1: Pre-operative appearance of thegrowth in the posterior tongue 
The growth was clinically diagnosed to be a Traumatic fibroma. The lingual ectopic thyroid gland, Haemangioma, Giant cell fibroma, To evaluate the extent of the lesion, an MRI scan was done. The MRI revealed no indurations of the underlying tissues and absence of extension into the adjacent structures. Since, the location of the growth resembled that of a lingual thyroid, investigations were done to rule out the possibility. Radioactive Iodine uptake studies and Tc99 scan indicated the absence of an ectopic thyroid gland. Based on history, clinical examination and investigations, a final diagnosis of Traumatic Fibroma in relation to the posterior tongue was made. Complete excision of the lesion under general anaesthesia was planned. Prior to the surgery, routine haematological examination, urinary examination and a CT scan of the Chest were carried out as
Lipoma, Granular cell tumour, Osseous Choristoma and salivary gland tumour was the Differential diagnosis.

per the protocol for cases to be done under general anaesthesia. All the parameters were found to be within the normal range. The lesion was completely excised under GA (Figure 2). The excised lesion was sent for histological examination (Figure 3). The biopsy report revealed squamous epithelium with a circumscribed lesion of increased cellularity, composed of numerous thin-walled capillaries and dense collagen fibres (Figure 4). These features were suggestive of Traumatic Fibroma, thus confirming our diagnosis. The patient has recalled after 15 days and post-operative healing was uneventful (Figure 5). On a 2 year follow up, no signs of relapse were observed.

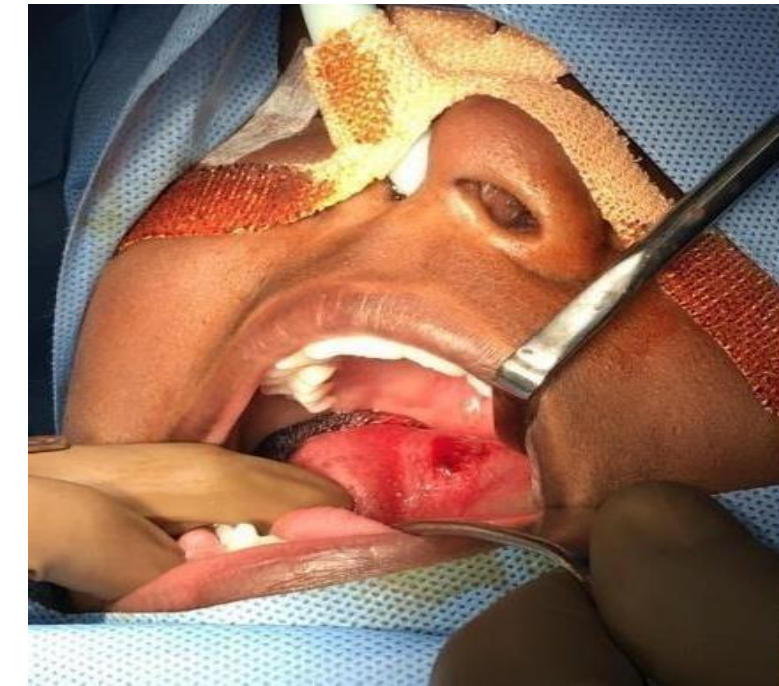

Figure 2: Intra-operative photograph

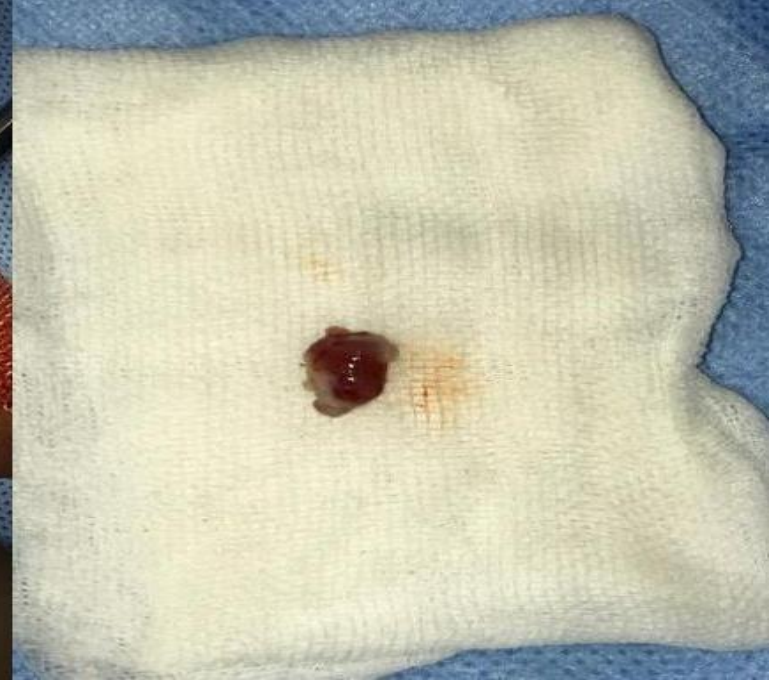

Figure 3: Excised lesion.

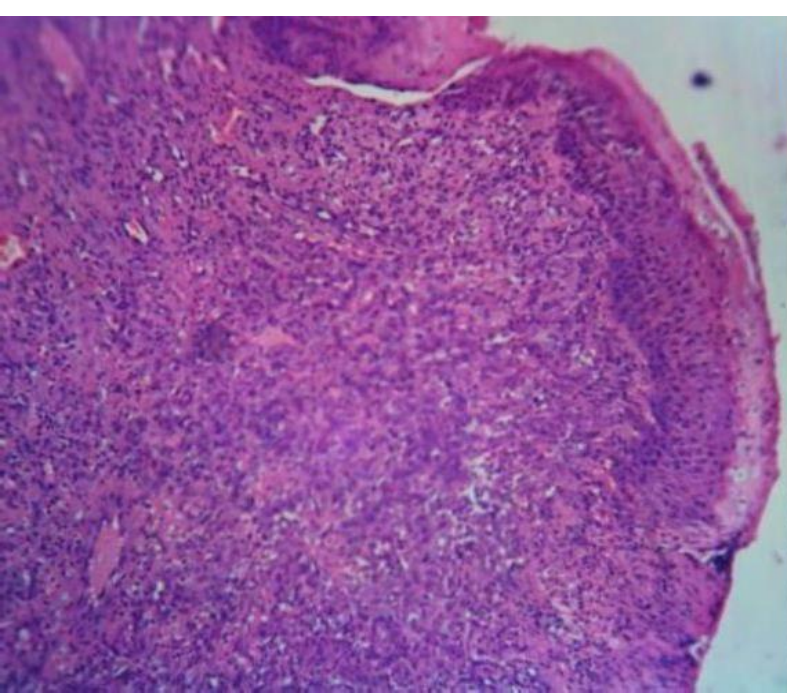

Figure 4: Histopathological image.

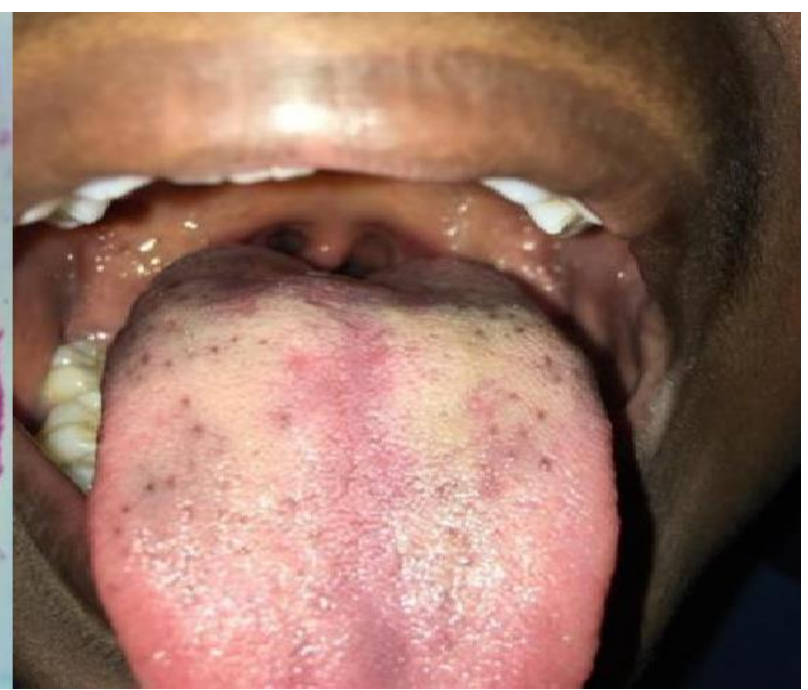

Figure 5: Post-operative photographafter 15 days.

\section{Discussion}

Fibroma is the most frequently occurring benign soft tissue neoplasm of the oral cavity. It is of connective tissue origin. It is generally preceded by either trauma or chronic irritation [4] that causes a reactive hyperplastic lesion. The tissues of the oral cavity react to the trauma and in response an unruly repair process begins. As a result, excessive fibrous connective tissue is produced, and it leads to the formation of a nodule or mass [5]. In the case reported, trauma to the tongue during toothbrushing was identified to be the etiology. Majority of the cases affected are seen in the third to fifth decade and more commonly in females than males.

Oral traumatic fibroma presents as an asymptomatic, well- circumscribed, solitary, dome- shaped swelling. It may be pedunculated or sessile [6]. They increase in size over a few weeks or months to reach its maximum size, generally not greater than $1 \mathrm{~cm}$ in diameter. Its surface may be smooth or ulcerated due to repetitive trauma. The commonest sites include the buccal mucosa, tongue, gingiva and lips [7].

Histologically, fibroma consists of bundles of interlacing collagen fibres interspersed with varying number of fibroblasts and small blood vessels. The overlying epithelium may be ulcerated, thinned or thickened [1]. A combination of history, clinical findings and histopathology will help to arrive at the diagnosis of fibroma. Biopsy 
is done to exclude otherconditions and confirm the diagnosis.

According to Barker and Lucas, traumatic fibromas exhibit a pattern of collagen arrangement depending on the site of the lesion and the amount of irritation. There are two types of patterns: (a) radiating pattern and (b) circular pattern. The authors hypothesized that when there is a greater degree of trauma, radiating pattern appears in sites that are immobile in nature (e.g., palate), while lesser trauma induces the circular pattern which occurs in sites that are flexible in nature (e.g., cheeks) [8].

In 1986, 23,616 white persons over 35 years of age were screened by Bouquot \&Gundlach. It was determined that traumatic/ irritational fibroma was the most commonly occurring soft tissue lesion of the oral cavity. Fibromas accounted for $35.8 \%$ of all benign soft tissue

\section{Conclusion}

Fibromas are the most commonly occurring lesions in the oral cavity and hence extensive research has been done about the same. Though benign, proper history taking, clinical examination and histological investigation are of utmost importance to properly diagnose the lesion

\section{Declaration of Patient Consent}

The authors certify that we have obtained an appropriate patient consent form. In the form, the patient has given his consent for his images and other clinical information to be reported in the journal.

\section{References}

1. Shafer's Textbook of Oral Pathology, 7th edition, Ch. 2 - Benign and MalignantTumors of the Oral Cavity.

2. Rathva VJ (2013) "Traumatic fibroma of tongue". BMJ Case Reports. 2013 : b c r 2012008220 .

3. Phore S, Panchal RS (2018) "Traumatic oral lesions: Pictorial essay”. Med J DY PatilVidyapeeth. 11(2): 94-8.

4. de Santana Santos T, Martins-Filho PR, Piva MR, de Souza Andrade ES ( 201014 ) "Focal fibrous hyperplasia: A review of 193 cases”. J Oral Maxillofac Pathol. 18(Suppl 1): S86-9.

5. Esmelli T, Lozada-Nur F, Epstein J (2005) "Common benign oral soft tissue masses”. DentClin North Am. 49(1): 223-40.

6. Borkar P, Gattani D, Uike S (2019) "Traumatic Fibroma - A CaseReport”. J Clin Case Rep. 2(2): 1021

7. Vujhini SK, Sridhar Reddy E, Sudheer MVS, Katikaneni HK (2016) "Irritation fibroma of the tongue: A case report". Int massesdiagnosed among the study population [9]. Similarly, in 2008, Santiago Torres Domingo et al, undertook a study to identify the most common benign tumours of the oral mucosa. Among the population studied, 300 patients were diagnosed with a benign tumour in the oral cavity and $153(53.3 \%)$ out of the 300 were histologically diagnosed as a fibroma. Thus, it was concluded that fibroma is the most frequent benign tumour of the oral cavity [10].

The treatment plan consists of conservative surgical excision and management of the etiology. The lesion usually does not recur, unless incompletely removed and has no malignant transformation [11]. In addition to the conventional surgical scalpel method, laser and electrocautery are newer treatment modalities that have added advantages [12].

and formulate the treatment plan. Accurate diagnosis, treatment and elimination of the irritant play an important role in preventing the recurrence of the growth

The patient understands that his name and initials will not be published, and due efforts will be made to conceal his identity, but anonymity cannot be guaranteed.

J Res Med Sci. 4(4): 1272-1273

8. Barker DS, Lucas RB (1967) "Localised fibrous overgrowths of the oral mucosa". Br J OralSurg 5(2): 86-92.

9. Bouquot JE, Gundlach KK (1986) "Oral exophytic lesions in 23,616 white Americans over 35 years of age”. Oral Surg Oral Med Oral Pathol. 62(3): 284-291.

10. Torres-Domingo S, Bagan JV, Jiménez Y, Poveda R, Murillo J, et al. (2008) "Benign tumors of the oral mucosa: A study of 300 patients". Med Oral Patol Oral Cir Bucal. 13(3): E161-6.

11. Sachdeva A, Bhateja S, Arora G (2018) "Traumatic fibroma: a case series". Journal of Dental Sciences. 5(1).

12. Lulla RV, Jaiswal P ( 2020 ) “Traumatic Fibroma: A Case Report". European Journal of Molecular \& Clinical Medicine. 7(7): 1653-1660 